\title{
18. MID- TO LATE QUATERNARY STABLE ISOTOPIC STRATIGRAPHY AND PALEOCEANOGRAPHY AT SITE 919 IN THE IRMINGER BASIN ${ }^{1}$
}

\author{
Benjamin P. Flower ${ }^{2}$
}

\begin{abstract}
Stable isotopic records based on the planktonic foraminifer Neogloboquadrina pachyderma (sinistrally coiling) from Holes $919 \mathrm{~A}$ and $919 \mathrm{~B}$ from the western Irminger Basin $\left(62^{\circ} 40^{\prime} \mathrm{N}, 37^{\circ} 28^{\prime} \mathrm{W}\right)$ provide records of surface water environments of the mid- to late Quaternary in the Irminger Sea. The 147-m sequence clearly shows oxygen isotope Stages 1 through 20 (possibly to 25). Assignment of oxygen isotope stages is supported by calcareous nannofossil biostratigraphy, magnetic polarity stratigraphy, and strontium isotope stratigraphy. Sedimentation rates average $\sim 17.9 \mathrm{~cm} / \mathrm{k} . \mathrm{y}$. for the $960-420 \mathrm{ka}$ interval and $\sim 13.4 \mathrm{~cm} /$ k.y. for the $420-0 \mathrm{ka}$ interval. Significantly, interglacial $\delta^{18} \mathrm{O}$ in Stages 21 through 15 is $0.5 \%$ o to $0.8 \%$ o lower than in succeeding interglacials, suggesting warmer sea surface temperatures and/or lower salinity in this interval. The magnitude and rapidity of these low- $\delta^{18} O$ events suggest large variations in salinity of the Irminger Sea during the 860-600 ka interval, perhaps reflecting greater meltwater flux derived from the Greenland Ice Sheet.
\end{abstract}

\section{INTRODUCTION}

Site 919 was drilled at $2088.2 \mathrm{~m}$ water depth in the Irminger Basin $\left(62^{\circ} 40^{\prime} \mathrm{N}, 37^{\circ} 28^{\prime} \mathrm{W}\right)$. The site was designed to (1) recover an expanded Quaternary fossiliferous section and (2) provide information for future deep drilling into basement, in order to sample the transition from the seaward-dipping reflector series (SDRS) to normal oceanic crust on the southeast Greenland Margin. Situated downstream of the Denmark Strait just northeast of the Erik Drift, Site 919 (Fig. 1) yielded a continuous mid- to upper Quaternary sequence rich in calcareous and siliceous microfossils. Nearby, Site 918 yielded a lower Eocene to Holocene sequence, which documented, among other findings, ice-rafted material derived from Greenland from about $7 \mathrm{Ma}$ to the present (Larsen et al., 1994). At Site 919, high sedimentation rates (averaging $\sim 15.5 \mathrm{~cm} / \mathrm{k} . y . ;$ Ali and Vandamme, this volume) offer opportunities for very detailed, high-resolution paleoenvironmental studies in the Quaternary.

Planktonic foraminifers are generally common to abundant and preservation is generally good throughout the sequence, based on about two samples per section sampling (Spezzaferri and Spiegler, this volume). Here, stable isotopic data are reported on Neogloboquadrina pachyderma (sinistrally coiling [s.]) from the same samples. Stable isotopic stratigraphy, integrated with calcareous nannofossil biostratigraphy, magnetostratigraphy, and strontium isotopic stratigraphy, provides a temporal framework for lithologic and faunal studies on this sequence. Significantly, the data trace the history of surface water environments in the Irminger Sea during the mid- to late Quaternary. Also, oxygen isotopic stratigraphy is intercalibrated with calcareous nannofossil and diatom zonations, including calibration for the first time of two diatom biostratigraphic events in the late Quaternary of the North Atlantic (Koç and Flower, this volume).

These data also improve the geographic coverage of Quaternary $\delta^{18} \mathrm{O}$ and $\delta^{13} \mathrm{C}$ records from the Nordic Seas. Site 919 in the southern Greenland Sea complements existing records from the Iceland Sea (Site 907; Fronval and Jansen, 1996; McManus et al., 1996), the Norwegian Sea (Sites 643 and 644; Jansen et al., 1988; Fronval et al.,

${ }^{1}$ Saunders, A.D., Larsen, H.C., and Wise, S.W., Jr. (Eds.), 1998. Proc. ODP, Sci. Results, 152: College Station, TX (Ocean Drilling Program).

${ }^{2}$ Institute of Marine Sciences and Earth Sciences Board, University of California, Santa Cruz, Santa Cruz, CA 95064, U.S.A. flower@earthsci.ucsc.edu

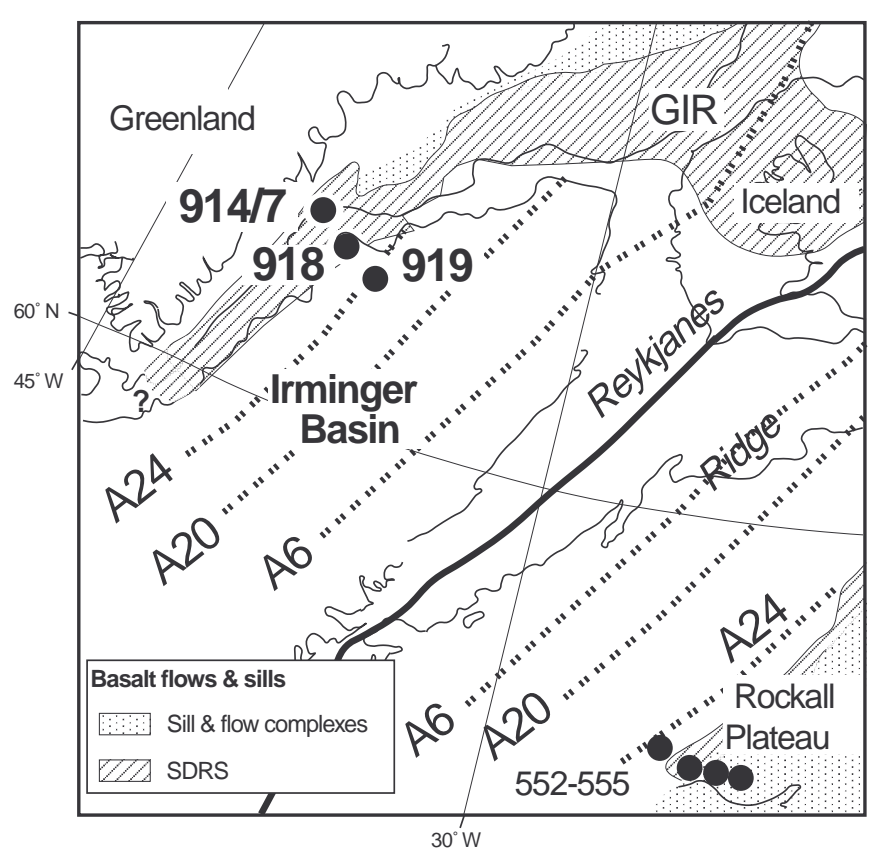

Figure 1. Location map showing Leg 152 drill sites in the Irminger Basin off the southeast Greenland Margin. Also shown are important ridges and basins in the area. Bathymetric contour interval is $500 \mathrm{~m}$. SDRS = seaward-dipping reflector sequence, and GIR = Greenland-Iceland Ridge.

1995; Fronval and Jansen, 1996), the Fram Strait (McManus et al., 1996; Bauch, in press), and from the Arctic Ocean (Flower, 1996; 1997). Detailed comparisons of regional $\delta^{18} \mathrm{O}$ and $\delta^{13} \mathrm{C}$ gradients during the Quaternary will now be possible.

\section{METHODS}

Samples were dried, weighed, soaked in a warm Calgon solution $\left(<50^{\circ} \mathrm{C}\right)$, washed over a $63-\mu \mathrm{m}$ sieve, dried, and weighed again. Individual planktonic foraminifers were handpicked from the 150 - to $250-\mu \mathrm{m}$ size fraction for isotopic analysis. Approximately 20 speci- 
mens of the planktonic foraminifer Neogloboquadrina pachyderma (s.) were used for each analysis.

Specimens for isotopic analysis were sonicated in methanol to remove adhering particles, roasted under vacuum at $375^{\circ} \mathrm{C}$, and reacted in $100 \%$ orthophosphoric acid at $90^{\circ} \mathrm{C}$. The evolved $\mathrm{CO}_{2}$ gas was analyzed on-line by a Fisons Prism isotope ratio mass spectrometer equipped with an automated preparation device in the laboratory of Christina Ravelo and James Zachos at the University of California, Santa Cruz. Instrumental precision based on NBS-19 and Carrera Marble carbonate standards run daily was better than $0.1 \%$ o for both $\delta^{18} \mathrm{O}$ and $\delta^{13} \mathrm{C}$. Sample precision based on replicate analyses of approximately $15 \%$ of the samples was $0.1 \%$ (mean standard deviation) for both $\delta^{18} \mathrm{O}$ and $\delta^{13} \mathrm{C}$. All isotopic data are expressed using standard $\delta$ notation in per mil relative to the Peedee belemnite (PDB) carbonate standard (Table 1).

\section{RESULTS}

Oxygen isotopic data based on Neogloboquadrina pachyderma (s.) exhibit a series of $1.3 \%$ variations from 0 to 90 meters below seafloor (mbsf), with values ranging from $2.7 \%$ o to $4.5 \%$ o. Amplitudes below 90 mbsf reach $1.8 \%$ and values range from $2.0 \%$ o to $4.2 \%$ o (Fig. 2). In addition, there are several sharp decreases in $\delta^{18} \mathrm{O}$ throughout the sequence, including excursions of $0.5 \%$ - $2.0 \%$ o marked by one or two data points at 21.46, 48.51, 50.98, 61.98, 91.03, and $120.94 \mathrm{mbsf}$ and three such excursions in the 127-133 mbsf interval.

Carbon isotopic values exhibit a series of $0.5 \% 0-1.0 \%$ variations from 0 to 90 mbsf, and range from $-0.7 \%$ o to $+0.5 \%$. Mean $\delta^{13} \mathrm{C}$ values are $0.5 \%$ lower below about 90 mbsf and exhibit a similar amplitude of $0.5 \%$ o $1.0 \%$ o. In addition, three distinct $\delta^{13} \mathrm{C}$ minima reaching $-1.6 \%$ o to $-2.0 \%$ o coincide with $\delta^{18} \mathrm{O}$ minima at $120.94,128.51$, and 132.12 mbsf (Fig. 2).

\section{DISCUSSION}

Oxygen and carbon isotopic data based on Neogloboquadrina pachyderma (s.) from Site 919 provide an isotopic stratigraphy and paleoenvironmental records of mid- to late Quaternary surface waters in the western Irminger Basin, East Greenland Margin. Almost 100\% core recovery allows generation of nearly continuous isotopic records from Holes 919A and 919B. Core 152-919B-3H was drilled starting at $90 \mathrm{mbsf}$ to overlap the lower part of Core 152-919A-10H by $3.5 \mathrm{~m}$. Numerous very distal turbidites are observed in Cores 152919A-1H through $3 \mathrm{H}$ and in $9 \mathrm{H}$ and $10 \mathrm{H}$; no turbidites were recorded in Hole 919B (Shipboard Scientific Party, 1994). The oxygen isotopic data largely reflect the well-known variations in global ice volume during Quaternary glacial-interglacial cycles (Shackleton and Opdyke, 1973; Imbrie et al., 1984).

\section{Age Control}

The $\delta^{18} \mathrm{O}$ data clearly show oxygen isotope Stages 1 through 20 (at $\sim 123 \mathrm{mbsf}$ and possibly to Stage 25 at $145 \mathrm{mbsf}$ ). Many of the oxygen isotope stages are easily distinguished and readily assigned, especially Stages 1-15. For example, Stage 5 features a minimum at $18.45 \mathrm{mbsf}$ inferred to correspond to Stage 5e. Interpretation of the isotope stratigraphy is aided by the last occurrence (LO) of Pseudoemiliania lacunosa at $60 \pm 0.75 \mathrm{mbsf}$ (Wei, this volume), which is independently dated at $458 \mathrm{ka}$ in Stage 12 (Thierstein et al., 1977).

In addition, recognition of the Brunhes/Matuyama boundary at approximately $120.5 \mathrm{mbsf}$ (Ali and Vandamme, this volume) provides an important age constraint and confirms that the $\delta^{18} \mathrm{O}$ minimum at 120.94 mbsf represents Stage 19. Further, strontium isotopic
Table 1. Stable isotopic data from Holes 919A and 919B.

\begin{tabular}{|c|c|c|c|c|}
\hline $\begin{array}{l}\text { Core, section, } \\
\text { interval }(\mathrm{cm})\end{array}$ & $\begin{array}{l}\text { Depth } \\
\text { (mbsf) }\end{array}$ & $\begin{array}{l}\text { Age } \\
(\mathrm{ka})\end{array}$ & $\begin{array}{c}\delta^{13} \mathrm{C} \\
(\% \circ \mathrm{PDB})\end{array}$ & $\begin{array}{c}\delta^{18} \mathrm{O} \\
(\% \circ \mathrm{PDB})\end{array}$ \\
\hline \multicolumn{5}{|l|}{ 152-919A- } \\
\hline $1 \mathrm{H}-1,9-11$ & 0.09 & 0.72 & -0.01 & 2.63 \\
\hline $1 \mathrm{H}-1,69-71$ & 0.69 & 5.52 & 0.08 & 3.27 \\
\hline $1 \mathrm{H}-1,130-132$ & 1.30 & 10.40 & -0.30 & 2.70 \\
\hline $1 \mathrm{H}-2,20-22$ & 1.70 & 13.38 & -0.13 & 4.49 \\
\hline $1 \mathrm{H}-2,96-98$ & 2.46 & 18.62 & -0.21 & 3.45 \\
\hline $1 \mathrm{H}-2,130-132$ & 2.80 & 20.97 & -0.20 & 3.69 \\
\hline $1 \mathrm{H}-3,20-22$ & 3.20 & 23.73 & 0.03 & 4.20 \\
\hline $1 \mathrm{H}-3,74-78$ & 3.76 & 27.60 & 0.02 & 4.25 \\
\hline $1 \mathrm{H}-3,96-98$ & 3.96 & 28.98 & 0.00 & 3.84 \\
\hline $1 \mathrm{H}-3,130-132$ & 4.30 & 31.32 & 0.20 & 4.03 \\
\hline $1 \mathrm{H}-4,20-22$ & 4.70 & 34.08 & 0.27 & 3.65 \\
\hline $1 \mathrm{H}-4,130-132$ & 5.80 & 41.67 & 0.20 & 3.94 \\
\hline $1 \mathrm{H}-5,10-14$ & 6.10 & 43.74 & 0.19 & 3.65 \\
\hline $1 \mathrm{H}-5,10-14$ & 6.10 & 43.74 & -0.49 & 2.78 \\
\hline $1 \mathrm{H}-5,20-22$ & 6.20 & 44.43 & 0.15 & 3.96 \\
\hline $1 \mathrm{H}-5,97-99$ & 6.97 & 49.75 & 0.26 & 3.84 \\
\hline $1 \mathrm{H}-5,130-132$ & 7.30 & 52.02 & 0.33 & 3.61 \\
\hline $2 \mathrm{H}-2,14-16$ & 9.64 & 68.17 & 0.00 & 3.96 \\
\hline $2 \mathrm{H}-2,95-97$ & 10.45 & 73.58 & -0.02 & 3.30 \\
\hline $2 \mathrm{H}-3,13-15$ & 11.13 & 77.96 & -0.06 & 3.28 \\
\hline $2 \mathrm{H}-3,95-97$ & 11.95 & 83.24 & -0.20 & 3.24 \\
\hline $2 \mathrm{H}-4,14-16$ & 12.64 & 87.68 & -0.40 & 3.17 \\
\hline $2 \mathrm{H}-4,78-92$ & 13.34 & 92.19 & -0.08 & 3.83 \\
\hline $2 \mathrm{H}-4,101-103$ & 13.51 & 93.28 & -0.15 & 3.59 \\
\hline $2 \mathrm{H}-5,13-15$ & 14.13 & 97.28 & 0.34 & 3.42 \\
\hline $2 \mathrm{H}-5,94-96$ & 14.94 & 102.49 & 0.62 & 3.63 \\
\hline $2 \mathrm{H}-5,120-122$ & 15.20 & 104.17 & 0.47 & 3.26 \\
\hline $2 \mathrm{H}-6,13-15$ & 15.63 & 106.94 & 0.38 & 3.17 \\
\hline $3 \mathrm{H}-1,8-10$ & 17.58 & 119.50 & 0.08 & 2.96 \\
\hline $3 \mathrm{H}-1,95-97$ & 18.45 & 125.10 & 0.06 & 2.92 \\
\hline $3 \mathrm{H}-1,119-123$ & 18.69 & 126.65 & -0.09 & 3.08 \\
\hline $3 \mathrm{H}-2,8-10$ & 19.08 & 129.38 & -0.16 & 4.11 \\
\hline $3 \mathrm{H}-2,30-32$ & 19.30 & 131.06 & -0.34 & 4.02 \\
\hline $3 \mathrm{H}-2,95-97$ & 19.95 & 136.03 & -0.42 & 4.09 \\
\hline $3 \mathrm{H}-3,8-10$ & 20.58 & 140.84 & -0.26 & 4.28 \\
\hline $3 \mathrm{H}-3,30-32$ & 20.80 & 142.53 & -0.40 & 4.11 \\
\hline $3 \mathrm{H}-3,96-98$ & 21.46 & 147.57 & -0.60 & 3.31 \\
\hline $3 \mathrm{H}-4,8-10$ & 22.08 & 152.31 & -0.39 & 3.92 \\
\hline $3 \mathrm{H}-4,96-98$ & 22.96 & 159.04 & -0.34 & 3.94 \\
\hline $3 \mathrm{H}-5,8-10$ & 23.58 & 163.78 & -0.41 & 4.17 \\
\hline $3 \mathrm{H}-5,43-47$ & 23.95 & 166.61 & -0.43 & 4.26 \\
\hline $3 \mathrm{H}-5,97-99$ & 24.47 & 170.58 & -0.57 & 3.46 \\
\hline $3 \mathrm{H}-6,7-9$ & 25.07 & 175.17 & -0.36 & 3.54 \\
\hline $3 \mathrm{H}-6,95-97$ & 25.95 & 181.90 & -0.36 & 3.52 \\
\hline $3 \mathrm{H}-6,120-124$ & 26.22 & 183.96 & -0.30 & 3.44 \\
\hline $3 \mathrm{H}-7,8-10$ & 26.58 & 186.71 & -0.16 & 3.39 \\
\hline $4 \mathrm{H}-1,47-49$ & 27.47 & 193.52 & -0.06 & 3.35 \\
\hline $4 \mathrm{H}-1,87-89$ & 27.87 & 196.58 & -0.20 & 4.03 \\
\hline $4 \mathrm{H}-1,130-132$ & 28.30 & 199.86 & -0.29 & 3.10 \\
\hline $4 \mathrm{H}-2,95-97$ & 29.45 & 208.65 & -0.03 & 3.48 \\
\hline $4 \mathrm{H}-3,23-27$ & 30.25 & 214.77 & -0.05 & 3.74 \\
\hline $4 \mathrm{H}-3,68-70$ & 30.68 & 218.06 & 0.08 & 3.28 \\
\hline $4 \mathrm{H}-5,95-97$ & 33.95 & 243.06 & -0.62 & 3.45 \\
\hline $4 \mathrm{H}-6,24-28$ & 34.76 & 249.25 & -0.22 & 3.52 \\
\hline $4 \mathrm{H}-6,95-97$ & 35.45 & 254.52 & -0.48 & 3.76 \\
\hline $5 \mathrm{H}-1,95-97$ & 37.45 & 269.81 & -0.49 & 3.70 \\
\hline $5 \mathrm{H}-2,95-97$ & 38.95 & 281.28 & -0.69 & 3.76 \\
\hline $5 \mathrm{H}-3,73-77$ & 40.25 & 291.22 & -0.57 & 3.94 \\
\hline $5 \mathrm{H}-3,93-95$ & 40.43 & 292.60 & -0.26 & 3.78 \\
\hline $5 \mathrm{H}-4,97-99$ & 41.97 & 304.37 & -0.25 & 3.17 \\
\hline $5 \mathrm{H}-6,95-97$ & 44.95 & 327.15 & -0.14 & 3.09 \\
\hline $5 \mathrm{H}-7,45-47$ & 45.95 & 334.80 & 0.19 & 2.94 \\
\hline $6 \mathrm{H}-1,48-50$ & 46.48 & 338.85 & -0.79 & 4.14 \\
\hline $6 \mathrm{H}-1,97-99$ & 46.97 & 342.95 & -0.52 & 3.84 \\
\hline $6 \mathrm{H}-1,148-150$ & 47.48 & 347.23 & -0.41 & 3.98 \\
\hline $6 \mathrm{H}-2,48-50$ & 47.98 & 351.43 & -0.37 & 3.59 \\
\hline $6 \mathrm{H}-2,93-95$ & 48.43 & 355.21 & -0.44 & 3.25 \\
\hline $6 \mathrm{H}-2,97-99$ & 48.47 & 355.55 & -0.43 & 3.24 \\
\hline $6 \mathrm{H}-3,48-50$ & 49.48 & 364.03 & -0.02 & 3.72 \\
\hline $6 \mathrm{H}-3,97-99$ & 49.97 & 368.15 & 0.10 & 3.63 \\
\hline $6 \mathrm{H}-4,48-50$ & 50.98 & 376.63 & -0.14 & 3.16 \\
\hline $6 \mathrm{H}-4,128-130$ & 51.78 & 383.35 & 0.39 & 4.11 \\
\hline $6 \mathrm{H}-5,48-50$ & 52.48 & 389.23 & 0.39 & 3.82 \\
\hline $6 \mathrm{H}-5,97-99$ & 52.97 & 393.35 & -0.23 & 2.83 \\
\hline $6 \mathrm{H}-5,99-103$ & 53.01 & 393.68 & -0.46 & 2.99 \\
\hline $6 \mathrm{H}-5,148-150$ & 53.48 & 397.63 & 0.13 & 3.22 \\
\hline $6 \mathrm{H}-6,28-30$ & 53.78 & 400.15 & 0.30 & 3.11 \\
\hline $6 \mathrm{H}-6,78-80$ & 54.28 & 404.35 & 0.29 & 3.04 \\
\hline $6 \mathrm{H}-7,48-50$ & 55.98 & 418.63 & -0.14 & 3.84 \\
\hline $7 \mathrm{H}-1,48-50$ & 55.98 & 418.63 & -0.06 & 4.39 \\
\hline $7 \mathrm{H}-1,95-99$ & 56.47 & 422.75 & -0.60 & 3.41 \\
\hline $7 \mathrm{H}-1,148-150$ & 56.98 & 425.31 & -0.17 & 4.60 \\
\hline $7 \mathrm{H}-2,48-50$ & 57.48 & 427.71 & -0.30 & 3.90 \\
\hline $7 \mathrm{H}-2,95-97$ & 57.95 & 429.97 & -0.44 & 3.75 \\
\hline $7 \mathrm{H}-2,148-150$ & 58.48 & 432.52 & -0.44 & 4.05 \\
\hline $7 \mathrm{H}-3,28-30$ & 58.78 & 433.97 & -0.34 & 4.05 \\
\hline $7 \mathrm{H}-3,48-50$ & 58.98 & 434.93 & -0.37 & 3.49 \\
\hline $7 \mathrm{H}-3,88-90$ & 59.38 & 436.85 & 0.03 & 4.11 \\
\hline
\end{tabular}


Table 1 (continued).

\begin{tabular}{|c|c|c|c|c|}
\hline $\begin{array}{l}\text { Core, section, } \\
\text { interval }(\mathrm{cm})\end{array}$ & $\begin{array}{l}\text { Depth } \\
\text { (mbsf) }\end{array}$ & $\begin{array}{l}\text { Age } \\
\text { (ka) }\end{array}$ & $\begin{array}{c}\delta^{13} \mathrm{C} \\
(\% \circ \mathrm{PDB})\end{array}$ & $\begin{array}{c}\delta^{18} \mathrm{O} \\
(\% \circ \mathrm{PDB})\end{array}$ \\
\hline $7 \mathrm{H}-3,128-130$ & 59.78 & 438.78 & -0.03 & 3.88 \\
\hline $7 \mathrm{H}-4,48-50$ & 60.48 & 442.14 & -0.56 & 4.04 \\
\hline $7 \mathrm{H}-4,97-99$ & 60.97 & 444.50 & -0.38 & 3.31 \\
\hline $7 \mathrm{H}-4,148-150$ & 61.48 & 446.95 & -0.32 & 4.28 \\
\hline $7 \mathrm{H}-5,44-52$ & 61.98 & 449.36 & 0.08 & 2.65 \\
\hline $7 \mathrm{H}-5,95-97$ & 62.45 & 451.62 & -0.39 & 3.30 \\
\hline $7 \mathrm{H}-5,148-150$ & 63.98 & 458.98 & 0.28 & 3.81 \\
\hline $7 \mathrm{H}-6,94-96$ & 63.94 & 458.78 & 0.18 & 3.10 \\
\hline $8 \mathrm{H}-1,79-81$ & 65.79 & 467.68 & 0.10 & 2.92 \\
\hline $8 \mathrm{H}-2,8-12$ & 66.60 & 471.58 & 0.09 & 3.16 \\
\hline $8 \mathrm{H}-3,96-98$ & 68.96 & 482.93 & 0.21 & 2.94 \\
\hline $8 \mathrm{H}-4,96-98$ & 70.46 & 490.14 & 0.12 & 3.15 \\
\hline $8 \mathrm{H}-5,96-98$ & 71.96 & 497.36 & 0.19 & 2.99 \\
\hline $8 \mathrm{H}-6,96-98$ & 73.46 & 504.57 & -0.10 & 2.86 \\
\hline $8 \mathrm{H}-6,138-142$ & 73.90 & 506.69 & -0.25 & 2.73 \\
\hline $9 \mathrm{H}-1,30-32$ & 74.80 & 511.01 & -0.65 & 2.72 \\
\hline $9 \mathrm{H}-2,29-31$ & 76.29 & 518.18 & -0.20 & 3.12 \\
\hline $9 \mathrm{H}-2,130-132$ & 77.30 & 523.04 & -0.34 & 3.18 \\
\hline $9 \mathrm{H}-3,29-31$ & 77.79 & 525.54 & -0.22 & 3.27 \\
\hline $9 \mathrm{H}-3,45-49$ & 77.97 & 526.49 & -0.40 & 3.76 \\
\hline $9 \mathrm{H}-3,130-132$ & 78.80 & 530.90 & -0.14 & 3.97 \\
\hline $9 \mathrm{H}-4,29-31$ & 79.29 & 533.49 & -0.25 & 4.07 \\
\hline $9 \mathrm{H}-4,130-132$ & 80.30 & 538.85 & -0.57 & 3.67 \\
\hline $9 \mathrm{H}-5,30-32$ & 80.80 & 541.50 & -0.61 & 3.62 \\
\hline $9 \mathrm{H}-5,129-131$ & 81.79 & 546.75 & -0.59 & 3.69 \\
\hline $9 \mathrm{H}-6,112-116$ & 83.14 & 553.91 & -0.40 & 3.52 \\
\hline $9 \mathrm{H}-6,129-131$ & 83.29 & 554.71 & -0.72 & 3.59 \\
\hline $9 \mathrm{H}-7,29-31$ & 83.79 & 557.36 & -0.51 & 4.05 \\
\hline $10 \mathrm{H}-1,46-48$ & 84.46 & 560.91 & -0.47 & 3.78 \\
\hline $10 \mathrm{H}-1,118-120$ & 85.18 & 564.73 & -0.33 & 4.05 \\
\hline $10 \mathrm{H}-2,36-38$ & 85.86 & 568.34 & -0.46 & 3.25 \\
\hline $10 \mathrm{H}-2,88-90$ & 86.38 & 571.10 & -0.45 & 2.94 \\
\hline $10 \mathrm{H}-3,35-37$ & 87.35 & 576.24 & -0.37 & 3.10 \\
\hline $10 \mathrm{H}-3,79-83$ & 87.81 & 578.68 & -0.49 & 2.96 \\
\hline $10 \mathrm{H}-3,141-143$ & 88.41 & 581.87 & -0.40 & 3.49 \\
\hline $10 \mathrm{H}-4,37-39$ & 88.87 & 584.30 & -0.86 & 2.94 \\
\hline $10 \mathrm{H}-5,70-72$ & 90.70 & 594.01 & -0.42 & 2.58 \\
\hline $10 \mathrm{H}-5,103-105$ & 91.03 & 595.76 & -0.52 & 2.09 \\
\hline $10 \mathrm{H}-5,103-105$ & 91.03 & 595.76 & -0.64 & 1.96 \\
\hline $10 \mathrm{H}-6,41-43$ & 91.91 & 600.43 & -0.65 & 3.90 \\
\hline $10 \mathrm{H}-6,41-43$ & 91.91 & 600.43 & -0.70 & 4.16 \\
\hline $10 \mathrm{H}-6,46-60$ & 92.03 & 601.07 & -0.61 & 3.75 \\
\hline \multicolumn{5}{|l|}{ 152-919B- } \\
\hline $3 \mathrm{H}-1,94-96$ & 90.94 & 595.28 & -0.57 & 3.69 \\
\hline $3 \mathrm{H}-2,100-102$ & 92.50 & 603.56 & -0.14 & 3.11 \\
\hline $3 \mathrm{H}-2,124-128$ & 92.76 & 604.94 & -0.27 & 3.08 \\
\hline $3 \mathrm{H}-3,95-97$ & 93.95 & 611.25 & -0.83 & 2.06 \\
\hline $3 \mathrm{H}-4,96-98$ & 95.46 & 619.26 & -0.92 & 2.50 \\
\hline $3 \mathrm{H}-4,132-136$ & 95.82 & 621.53 & -0.67 & 4.17 \\
\hline $3 \mathrm{H}-5,95-97$ & 96.95 & 629.37 & -1.02 & 3.80 \\
\hline $3 \mathrm{H}-6,96-98$ & 98.46 & 639.85 & -0.65 & 3.93 \\
\hline $3 \mathrm{H}-6,120-124$ & 99.70 & 648.46 & -1.30 & 2.63 \\
\hline $3 \mathrm{H}-\mathrm{CC}, 8-9$ & 99.68 & 648.32 & -0.97 & 3.67 \\
\hline $4 \mathrm{H}-1,91-95$ & 100.41 & 653.38 & -1.12 & 3.61 \\
\hline $4 \mathrm{H}-1,95-97$ & 100.45 & 653.66 & -1.19 & 3.47 \\
\hline $4 \mathrm{H}-2,95-97$ & 101.95 & 664.07 & -1.00 & 3.86 \\
\hline $4 \mathrm{H}-3,95-97$ & 103.45 & 674.48 & -0.54 & 3.69 \\
\hline $4 \mathrm{H}-3,134-135$ & 103.84 & 677.19 & -0.77 & 2.71 \\
\hline $4 \mathrm{H}-4,28-30$ & 104.28 & 680.24 & -0.45 & 2.85 \\
\hline $4 \mathrm{H}-4,48-50$ & 104.48 & 681.63 & -1.09 & 2.70 \\
\hline $4 \mathrm{H}-4,88-92$ & 104.88 & 684.41 & -0.70 & 2.29 \\
\hline $4 \mathrm{H}-4,128-130$ & 105.28 & 687.18 & -1.39 & 2.71 \\
\hline $4 \mathrm{H}-5,95-97$ & 106.45 & 695.30 & -0.53 & 2.54 \\
\hline $4 \mathrm{H}-7,21-25$ & 108.71 & 710.99 & -0.74 & 2.86 \\
\hline $5 \mathrm{H}-1,28-30$ & 109.28 & 714.63 & -0.24 & 4.04 \\
\hline $5 \mathrm{H}-1,95-97$ & 109.95 & 718.53 & -0.29 & 3.87 \\
\hline $5 \mathrm{H}-2,44-45$ & 110.94 & 724.30 & -0.53 & 3.39 \\
\hline $5 \mathrm{H}-2,81-85$ & 111.31 & 726.46 & -0.04 & 3.63 \\
\hline $5 \mathrm{H}-3,95-97$ & 112.95 & 736.01 & -0.51 & 3.46 \\
\hline $5 \mathrm{H}-4,94-96$ & 114.44 & 744.69 & -0.66 & 3.02 \\
\hline $5 \mathrm{H}-5,77-81$ & 115.77 & 752.44 & -0.85 & 3.82 \\
\hline $5 \mathrm{H}-5,97-99$ & 115.97 & 753.61 & -0.91 & 3.20 \\
\hline $5 \mathrm{H}-6,97-99$ & 117.47 & 762.35 & -0.50 & 3.66 \\
\hline $6 \mathrm{H}-1,97-99$ & 119.47 & 774.00 & -0.78 & 2.66 \\
\hline $6 \mathrm{H}-2,78-82$ & 120.78 & 781.90 & -1.07 & 2.01 \\
\hline $6 \mathrm{H}-2,94-96$ & 120.94 & 782.99 & -1.52 & 2.00 \\
\hline $6 \mathrm{H}-3,96-98$ & 122.46 & 793.30 & -0.67 & 4.00 \\
\hline $6 \mathrm{H}-4,95-97$ & 123.95 & 803.41 & -0.12 & 3.83 \\
\hline $6 \mathrm{H}-5,124-128$ & 125.74 & 815.55 & -0.84 & 2.89 \\
\hline $6 \mathrm{H}-5,135-137$ & 125.85 & 816.30 & -0.92 & 3.10 \\
\hline $6 \mathrm{H}-6,58-60$ & 126.58 & 821.25 & -1.29 & 2.73 \\
\hline $6 \mathrm{H}-6,93-97$ & 126.93 & 823.62 & -1.17 & 2.96 \\
\hline $7 \mathrm{H}-1,21-23$ & 128.21 & 832.31 & -1.05 & 2.94 \\
\hline $7 \mathrm{H}-1,51-55$ & 128.51 & 834.34 & -1.97 & 2.03 \\
\hline $7 \mathrm{H}-1,51-55$ & 128.51 & 834.34 & -1.79 & 1.98 \\
\hline $7 \mathrm{H}-2,35-37$ & 129.85 & 843.43 & -1.02 & 3.73 \\
\hline $7 \mathrm{H}-2,112-114$ & 130.62 & 848.66 & -1.68 & 2.08 \\
\hline $7 \mathrm{H}-2,112-114$ & 130.62 & 848.66 & -1.39 & 2.49 \\
\hline $7 \mathrm{H}-2,129-131$ & 130.79 & 849.81 & -1.38 & 2.71 \\
\hline
\end{tabular}

Table 1 (continued).

\begin{tabular}{lcccc}
\hline $\begin{array}{c}\text { Core, section, } \\
\text { interval }(\mathrm{cm})\end{array}$ & $\begin{array}{c}\text { Depth } \\
(\mathrm{mbsf})\end{array}$ & $\begin{array}{c}\text { Age } \\
(\mathrm{ka})\end{array}$ & $\begin{array}{c}\delta^{13} \mathrm{C} \\
(\% \circ \mathrm{PDB})\end{array}$ & $\begin{array}{c}\delta^{18} \mathrm{O} \\
(\% \circ \mathrm{PDB})\end{array}$ \\
\hline 7H-3, 59-61 & 131.59 & 855.24 & -0.90 & 2.96 \\
7H-3,90-94 & 131.90 & 857.34 & -1.43 & 3.31 \\
7H-3, 90-94 & 131.90 & 857.34 & -1.27 & 3.08 \\
7H-3, 112-116 & 132.12 & 858.83 & -2.05 & 1.96 \\
7H-4, 30-32 & 132.80 & 863.45 & -0.87 & 3.81 \\
7H-4, 125-127 & 133.75 & 869.89 & -0.42 & 3.40 \\
7H-5, 125-127 & 135.25 & 880.07 & -0.38 & 3.62 \\
7H-6, 19-21 & 135.69 & 883.05 & -0.22 & 3.24 \\
7H-6, 143-145 & 136.93 & 891.47 & -0.56 & 2.96 \\
7H-7, 45-49 & 137.47 & 895.13 & -0.35 & 2.98 \\
8H-1, 56-58 & 138.06 & 899.13 & -0.59 & 3.59 \\
8H-1, 120-122 & 138.70 & 903.47 & -0.51 & 3.48 \\
8H-2, 29-31 & 139.29 & 907.48 & -0.78 & 3.35 \\
8H-2, 65-69 & 139.65 & 909.92 & -0.70 & 3.33 \\
8H-2, 138-140 & 140.38 & 914.87 & -0.92 & 3.05 \\
8H-3, 67-69 & 141.17 & 920.23 & -1.07 & 3.43 \\
8H-4, 9-11 & 142.09 & 926.47 & -0.75 & 3.75 \\
8H-4, 71-72 & 142.71 & 930.68 & -0.65 & 2.88 \\
8H-4, 128-130 & 143.28 & 934.55 & -1.05 & 3.02 \\
8H-5, 12-14 & 143.62 & 936.85 & -0.42 & 2.73 \\
8H-5, 108-110 & 144.58 & 943.37 & -0.26 & 3.21 \\
8H-6, 12-14 & 145.12 & 947.03 & -0.73 & 2.50 \\
8H-6, 109-111 & 146.09 & 953.59 & -0.81 & 2.44 \\
8H-7, 50-54 & 147.00 & 959.53 & -1.90 & 3.60 \\
8H-7, 50-54 & 147.00 & 959.53 & -1.24 & 3.37 \\
\hline
\end{tabular}

data confirm a late Quaternary age of <1.0 Ma for the entire Site 919 sequence (Israelson and Spezzaferri, this volume).

The age of the section below about $123 \mathrm{mbsf}$ is more difficult to assess using $\delta^{18} \mathrm{O}$ stratigraphy. However, strontium isotopic data (Israelson and Spezzaferri, this volume) and magnetic polarity data indicate an age of <1.0 Ma; the Jaramillo Chron is not reached (Ali and Vandamme, this volume). Accordingly, oxygen isotope Stages 21,23 , and 25 may be represented by low $\delta^{18} \mathrm{O}$ values from about 126 to $133 \mathrm{mbsf}, 137$ to $140 \mathrm{mbsf}$, and 143 to $146 \mathrm{mbsf}$, respectively. Large, rapid changes in $\delta^{18} \mathrm{O}$ superimposed on inferred Stage 21 from about 127 to 133 mbsf may primarily reflect salinity variations, as they are not associated with warm-water planktonic foraminifer faunas (Spezzaferri and Spiegler, this volume).

The $\delta^{18} \mathrm{O}$ record, supported by the $P$. lacunosa LO datum, the magnetic polarity record, and the strontium isotopic data, allows calculation of ages and sedimentation rates for at least the Brunhes Epoch. Stratigraphic datum levels plotted vs. age (Fig. 3) include the major oxygen isotope stage boundaries, the P. lacunosa LO datum, and the Brunhes/Matuyama Epoch boundary. Stage boundaries were dated according to an astronomically tuned time scale (Imbrie et al., 1984; Shackleton et al., 1990). Sedimentation rates average about $17.9 \mathrm{~cm} / \mathrm{k} . \mathrm{y}$. for the $960-420 \mathrm{ka}$ interval and about $13.4 \mathrm{~cm} / \mathrm{k} . \mathrm{y}$. for the $420-0$ ka interval.

\section{Surface Water Environments}

Based on the age control discussed in the preceding section, the $\delta^{18} \mathrm{O}$ data are plotted vs. age in Figure 4. A deep Pacific benthic record (Site 677; Shackleton and Hall, 1989) is compared to assess regional paleoenvironmental overprints in the Irminger Sea. The comparison illustrates that the dominant control of the Site $919 \delta^{18} \mathrm{O}$ signal is global ice volume. The amplitude of the $\delta^{18} \mathrm{O}$ signal from $\sim 600$ to $0 \mathrm{ka}$ is close to the $1.2 \%$ attributed to ice volume variations in the late Quaternary (Shackleton and Opdyke, 1973; Fairbanks, 1989; Shackleton and Hall, 1989). However, the $\delta^{18} \mathrm{O}$ amplitude may be underestimated due to undersampling of isotopic stages by relatively coarse resolution.

Significantly, there are large variations in $\delta^{18} \mathrm{O}$ of up to $2.1 \%$ in the interval from $\sim 860$ to $600 \mathrm{ka}$. Assuming an ice volume effect of $1.2 \%$ yields variations of up to $0.9 \%$, which probably reflects a combination of temperature and salinity changes. The rapidity and mag- 
Figure 2. Oxygen and carbon isotopic data based on Neogloboquadrina pachyderma (s.) plotted vs. depth in mbsf for Holes 919A and 919B, Irminger Basin. Also shown are inferred oxygen isotope stages and position of last occurrence (LO) of Pseudoemiliania lacunosa at $60 \pm 0.75$ mbsf. Core recovery and normal magnetic polarity are shown in black.
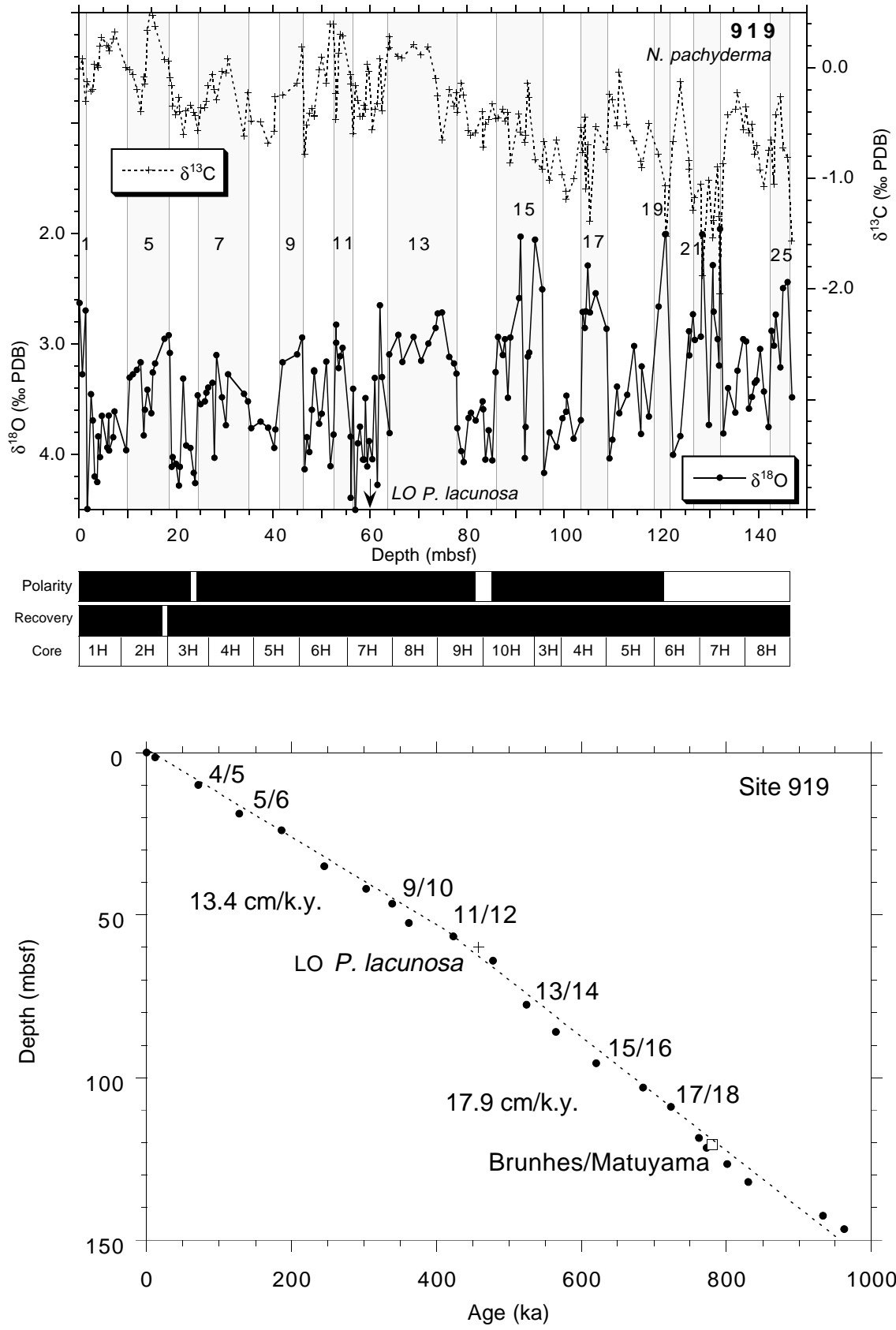

Figure 3. Age vs. depth plot of inferred oxygen isotope stage boundaries for Site 919. Stage boundary designations indicate those used in age model. Also shown are the LO of Pseudoemiliania lacunosa and the Brunhes/Matuyama boundary. Sedimentation rates average about $17.9 \mathrm{~cm} / \mathrm{k} . \mathrm{y}$. for the $960-420 \mathrm{ka}$ interval and about $13.4 \mathrm{~cm} / \mathrm{k} . \mathrm{y}$. for the $420-0 \mathrm{ka}$ interval. from the 127 to 133 mbsf interval, marked by large, rapid variations in $\delta^{18} \mathrm{O}$. Nor are the assemblages comparatively warmer prior to $\sim 600$ $\mathrm{ka}$; in fact the warmest assemblages (Sample 152-919A-6H-5, 97-99 $\mathrm{cm}$; Spezzaferri and Spiegler, this volume) are observed during Stage $11(\sim 420 \mathrm{ka})$. Thus the large variations in $\delta^{18} \mathrm{O}$ in the $860-600 \mathrm{ka}$ interval are largely attributed to salinity variations, with sea surface temperatures playing a smaller role. However, the relative importance of salinity and temperature changes still awaits independent evaluation by more detailed isotopic and quantitative micropaleontological studies.

Recent studies indicate that $N$. pachyderma (s.) indeed records the isotopic composition of the upper water column. N. pachyderma (s.) is shown to record the isotopic composition of the upper $30 \mathrm{~m}$ in the East Greenland Current (Johannessen et al., in press), while other studies suggest that $N$. pachyderma $\left(\right.$ s.) $\delta^{18} \mathrm{O}$ reflects the upper $200 \mathrm{~m}$ 

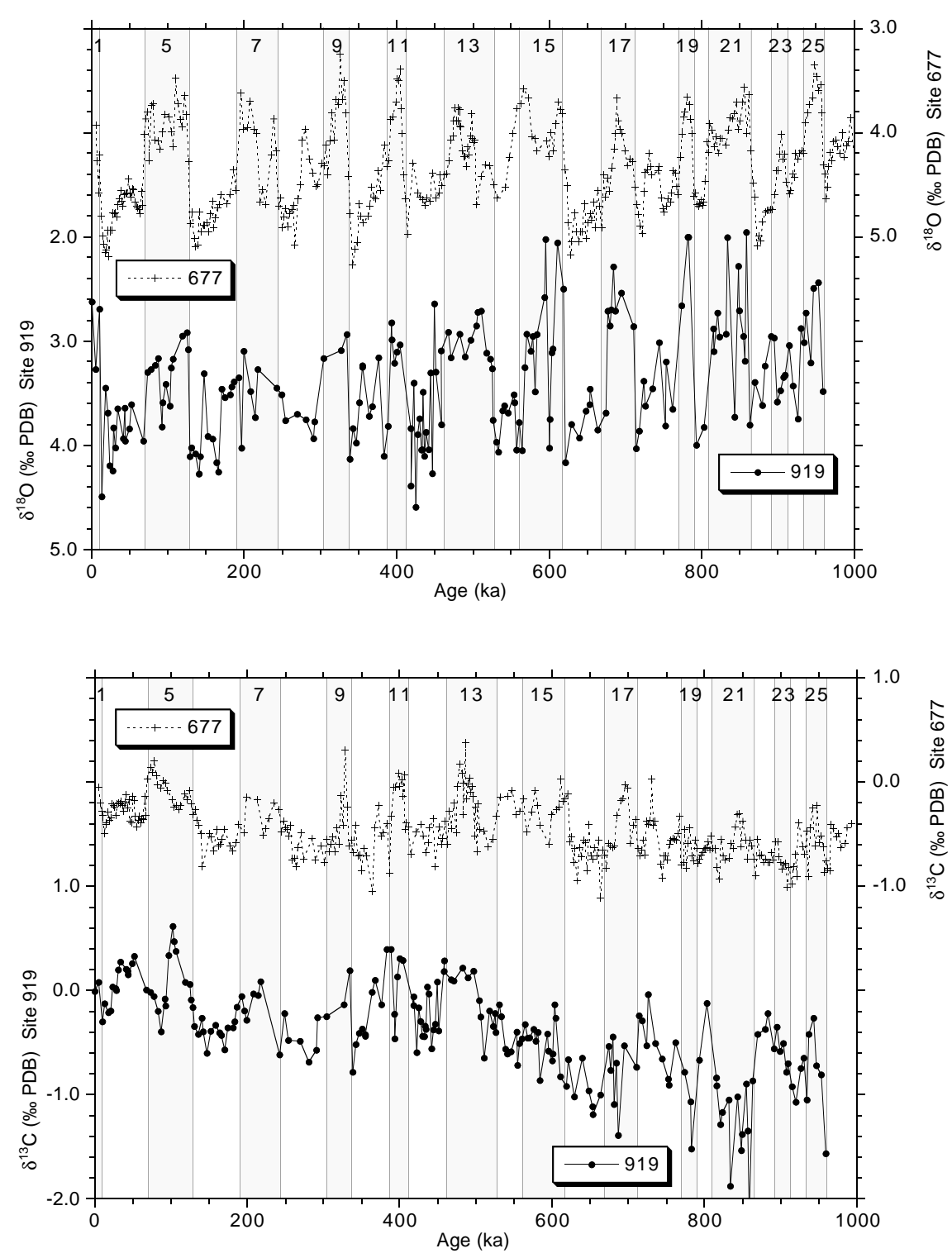

Figure 4. Oxygen isotopic records for Site 919 (based on Neogloboquadrina pachyderma [s.]) and for Site 677 (based mainly on Uvigerina; Shackleton and Hall, 1989) plotted vs. age. Also shown are oxygen isotope stages of Shackleton and Hall (1989).
Figure 5. Carbon isotopic records for Site 919 (based on Neogloboquadrina pachyderma [s.]) and for Site 677 (based mainly on Uvigerina; Shackleton and Hall, 1989) plotted vs. age. Also shown are oxygen isotope stages of Shackleton and Hall (1989).
(Kohfeld et al., 1994, 1996; Bauch et al., in press). Each study concludes that $N$. pachyderma (s.) accurately records the $\delta^{13} \mathrm{C}$ values of surface waters.

Comparison of $\delta^{13} \mathrm{C}$ records for Sites 919 and 677 (Fig. 5) also illustrates the global influence on the Site $919 \delta^{13} \mathrm{C}$ signal. The $\delta^{13} \mathrm{C}$ values generally covary, particularly from 550 to $0 \mathrm{ka}$. Site $919 \delta^{13} \mathrm{C}$ values diverge from Site 677 values prior to about $550 \mathrm{ka}$. Compared to the $550-0 \mathrm{ka}$ interval, Site $919 \delta^{13} \mathrm{C}$ values are about $0.5 \%$ lower and exhibit higher amplitudes prior to about $550 \mathrm{ka}$. These trends are also seen in Iceland Sea Site 907 (Fronval and Jansen, 1996), suggesting a regional change in the $\delta^{13} \mathrm{C}$ of Nordic Sea surface waters. A similar pattern for both $\delta^{18} \mathrm{O}$ and $\delta^{13} \mathrm{C}$ is observed on the Yermak Plateau, Arctic Ocean, where the amplitudes are even larger in the midQuaternary (Flower, 1996, 1997). Lower $\delta^{18} \mathrm{O}$ values and lower and more variable $\delta^{13} \mathrm{C}$ values were recorded by $N$. pachyderma (s.) prior to about $660 \mathrm{ka}$ at Site 910 on the Yermak Plateau. The similarities between these records point to common causes, perhaps involving large changes in surface salinities due to continental runoff prior to about $600 \mathrm{ka}$, with associated low $\delta^{13} \mathrm{C}$ of terrestrial organic matter. Greater variations in $\delta^{13} \mathrm{C}$ may reflect numerous changes in regional controls of surface water $\delta^{13} \mathrm{C}$, including upwelling, air-sea exchange, and terrestrial influx. However, the exact cause (or causes) remains unknown.

\section{Implications for North Atlantic Climate History}

Large and rapid changes in the $\delta^{18} \mathrm{O}$ of surface waters in the 860$600 \mathrm{ka}$ interval at Site 919 suggest large salinity changes of up to $0.9 \%$, as discussed above. Likely sources of low-salinity surface water in the western Irminger Sea include melting sea ice in the East Greenland Current and meltwater derived from the Greenland Ice Sheet. Either of these sources might indicate more variable meltwater influx during the 860-600 ka interval, perhaps related to greater instability of the Greenland Ice Sheet compared to the succeeding interval. The extent that this instability reflects external climate forcing or inherent instability of the Greenland Ice Sheet during this interval is unknown. A similar suggestion of greater instability of the circumArctic ice sheets including the Greenland Ice Sheet in the mid-Quaternary was made based on higher sedimentation rates for this interval on the Yermak Plateau (Leg 151 Shipboard Scientific Party, 
1995). Alternatively, more variable surface salinities in the Site 919 region may simply reflect changes in surface currents, involving increased advection of nearshore currents (with lower salinities) to the Irminger Basin.

Lower surface salinities in the East Greenland Current would greatly inhibit deep-water formation in key areas such as the Iceland Sea and the Labrador Sea, and might reduce North Atlantic Deep Water (NADW) production. It is interesting to note that the 900-600 ka interval featured suppression of NADW production during Stages 24-18 (Raymo et al., 1990) and some of the coldest glacial episodes of the Quaternary (Stages 22 and 16; Imbrie et al., 1984; Berger and Jansen, 1994). A potential link is meltwater-induced stratification of the water column, which would inhibit deep convection and formation of deep water (Rooth, 1982). This hypothesis can be tested by comparing indices for Denmark Strait overflow water during the mid- to late Quaternary, including benthic foraminifer $\delta^{13} \mathrm{C}$ and changes in grain-size distributions.

\section{SUMMARY}

Stable isotopic records $\left(\delta^{18} \mathrm{O}\right.$ and $\left.\delta^{13} \mathrm{C}\right)$ based on the planktonic foraminifer Neogloboquadrina pachyderma (s.) from Holes 919A and 919B provide an isotopic stratigraphy for the mid- to late Quaternary in the western Irminger Basin. The 147-m sequence clearly shows oxygen isotope Stages 1 through 20 (possibly to 25), with sedimentation rates averaging about $17.9 \mathrm{~cm} / \mathrm{k} . \mathrm{y}$. for the $960-420 \mathrm{ka}$ interval and about $13.4 \mathrm{~cm} / \mathrm{k} . \mathrm{y}$. for the $420-0 \mathrm{ka}$ interval. Assignment of oxygen isotope stages is supported by calcareous nannofossil biostratigraphy, magnetic polarity stratigraphy, and strontium isotope stratigraphy. Significantly, Stages 21 through 15 feature $\delta^{18} O$ changes of up to $2.1 \%$, suggesting some combination of meltwater influence and temperature variability in this interval. The magnitude and rapidity of these $\delta^{18} \mathrm{O}$ changes suggest large variations in meltwater influx to the Irminger Sea and perhaps instability of the Greenland Ice Sheet during the 860-600 ka interval.

\section{ACKNOWLEDGMENTS}

This work was supported by the JOI/USSSP (152-208526), with partial support from NSF (OPP-9423485 to B.P.F.), and by the USGS Climate Program. Stable isotope analyses were conducted in the laboratory of Christina Ravelo and James Zachos at the University of California, Santa Cruz. I thank J. Lynch-Stieglitz and Y. Wang for helpful reviews, S. Spezzaferri and T. Vallier for providing samples, and Mellany Manaig, Trang Le, and Bhavna Sahi for laboratory assistance.

\section{REFERENCES}

Bauch, D., Carstens, J., and Wefer, G., in press. Oxygen isotope composition of living Neogloboquadrina pachyderma (sin.) in the Arctic Ocean, Earth Planet. Sci. Lett.

Bauch, H.A., in press. Paleoceanography of the N. Atlantic Ocean $\left(68^{\circ}-\right.$ $78^{\circ} \mathrm{N}$ ) during the past $450 \mathrm{ky}$ deduced from planktic foraminiferal assemblages and stable isotopes. In Hass, H.C., and Kaminski, M.A. (Eds.), Micropaleontology and paleoceanography of the North Atlantic.

Berger, W.H., and Jansen, E., 1994. Mid-Pleistocene climate shift: the Nansen connection. In Johannessen, O.M., Muensch, R.D., and Overland, J.E. (Eds.), The Role of the Polar Oceans in Shaping the Global Environment. Geophys. Monogr., Am. Geophys. Union, 85:295-311.

Fairbanks, R.G., 1989. A 17,000-year glacio-eustatic sea level record: influence of glacial melting rates on the Younger Dryas event and deep-ocean circulation. Nature, 342:637-642.

Fairbanks, R.G., Charles, C.D., and Wright, J.D., 1992. Origin of global meltwater pulses. In Taylor, R.E., Long, A., and Kra, R.S. (Eds.), Radiocarbon after Four Decades: New York (Springer-Verlag).
Flower, B.P., 1996. Late Quaternary stable isotopic stratigraphy of Hole 910A, Yermak Plateau, Arctic Ocean: relations with Svalbard/Barents Sea ice-sheet history. In Thiede, J., Myhre, A.M., Firth, J.V., Johnson, G.L., and Ruddiman, W.F. (Eds.), Proc. ODP, Sci. Results, 151: College Station, TX (Ocean Drilling Program), 445-454.

Flower, B.P., 1997. Overconsolidated Section on the Yermak Plateau, Arctic Ocean: Ice Sheet Grounding Prior to 660 ka? Geology, 25:147-150.

Fronval, T., and Jansen, E., 1996. Late Neogene paleoclimates and paleoceanography in the Iceland-Norwegian Sea: evidence from the Iceland and Vøring Plateaus. In Thiede, J., Myhre, A.M., Firth, J.V., Johnson, G.L., and Ruddiman, W.F. (Eds.), Proc. ODP, Sci. Results, 151: College Station, TX (Ocean Drilling Program), 455-468.

Fronval, T., Jansen, E., Bloemendal, J., and Johnsen, S., 1995. Oceanic evidence for coherent fluctuations in Fennoscandian and Laurentide ice sheets on millennium timescales. Nature, 374:443-446.

Imbrie, J., Hays, J.D., Martinson, D.G., McIntyre, A., Mix, A.C., Morley, J.J., Pisias, N.G., Prell, W.L., and Shackleton, N.J., 1984. The orbital theory of Pleistocene climate: support from a revised chronology of the marine $\delta^{18} \mathrm{O}$ record. In Berger, A., Imbrie, J., Hays, J., Kukla, G., and Saltzman, B. (Eds.), Milankovitch and Climate (Pt. 1), NATO ASI Ser. C, Math Phys. Sci., 126: Dordrecht (Reidel), 269-305.

Jansen, E., Bleil, U., Henrich, R., Kringstad, L., and Slettemark, B., 1988. Paleoenvironmental changes in the Norwegian Sea and Northeast Atlantic during the last 2.8 m.y.: Deep Sea Drilling Project/Ocean Drilling Program Sites 610, 642, 643, and 644. Paleoceanography, 3:563-581.

Johannessen, T., Ravelo, A.C., and Jansen, E., in press. Distribution of carbon and oxygen isotopes in the Greenland, Iceland and Norwegian seas: relationship to water masses, nutrients and circulation. Mar. Geol.

Kohfeld, K.E., Fairbanks, R.G., and Smith, S.L., 1994. Decoupling of abundances and geochemical signals of $N$. pachyderma (s.) in marine sediments; evidence from MOCNESS plankton tows. Eos, 75:392.

Kohfeld, K.E., Fairbanks, R.G., Smith, S.L., and Walsh, I.D., 1996. Neogloboquadrina pachyderma (sinistral coiling) as paleoceanographic tracers in polar oceans: Evidence from Northeast Water Polynya plankton tows, sediment traps, and surface sediments. Paleoceanography, 11:679-699.

Larsen, H.C., Saunders, A.D., Clift, P.D., Beget, J., Wei, W., Spezzaferri, S., and the ODP Leg 152 Scientific Party, 1994. Seven million years of glaciation in Greenland. Science, 264:952-955.

Leg 151 Shipboard Scientific Party, 1995. Farthest north: ocean drilling in the Arctic Gateway region. GSA Today, 5:24-33.

McManus, J.F., Major, C.O., Flower, B.P., and Fronval, T., 1996. Variability in sea-surface conditions in the North Atlantic-Arctic Gateways during the last 140,000 years. In Thiede, J., Myhre, A.M., Firth, J.V., Johnson, G.L., and Ruddiman, W.F. (Eds.), Proc. ODP, Sci. Results, 151: College Station, TX (Ocean Drilling Program), 437-444.

Raymo, M.E., Ruddiman, W.F., Shackleton, N.J., and Oppo, D.W., 1990. Evolution of Atlantic-Pacific $\delta^{13} \mathrm{C}$ gradients over the last 2.5 m.y. Earth Planet. Sci. Lett., 97:353-368.

Rooth, C.G.H., 1982. Hydrology and ocean circulation. Progr. Oceanogr., $11: 131-149$.

Shackleton, N.J., Berger, A., and Peltier, W.A., 1990. An alternative astronomical calibration of the lower Pleistocene timescale based on ODP Site 677. Trans. R. Soc. Edinburgh: Earth Sci., 81:251-261.

Shackleton, N.J., and Hall, M.A., 1989. Stable isotope history of the Pleistocene at ODP Site 677. In Becker, K., Sakai, H., et al., Proc. ODP, Sci. Results, 111: College Station, TX (Ocean Drilling Program), 295-316.

Shackleton, N.J., and Opdyke, N.D., 1973. Oxygen isotope and paleomagnetic stratigraphy of equatorial Pacific core V28-238: oxygen isotope temperatures and ice volumes on a $10^{5}$ year and $10^{6}$ year scale. Quat. Res., 3:39-55.

Shipboard Scientific Party, 1994. Site 919. In Larsen, H.C., Saunders, A.D., Clift, P.D., et al., Proc. ODP, Init. Repts., 152: College Station, TX (Ocean Drilling Program), 257-277.

Thierstein, H.R., Geitzenauer, K., Molfino, B., and Shackleton, N.J., 1977. Global synchroneity of late Quaternary coccolith datum levels: validation by oxygen isotopes. Geology, 5:400-404.

Date of initial receipt: 4 November 1995

Date of acceptance: 29 May 1996

Ms 152SR-219 